\title{
Mutarotation and Ring Structure of Mannuronic Lactone
}

\author{
By Horace S. Isbell and Harriet L. Frush
}

\begin{abstract}
The numerous commercial applications of algin, a polysaccharide derived from certain marine plants, makes important the study of its principal constituent, D-mannuronic lactone. In this paper it is shown that by oxidation with bromine mannosaccharic dilactone of known structure is formed directly from mannuronic lactone without change in ring structure, and hence the latter substance, like mannosaccharic dilactone, has a bicyclic structure consisting of two butylene oxide rings. The initial rapid mutarotation reaction of the substance is show $\mathrm{n}$ to consist of a conversion of the beta furanose modification to an equilibrium mixture cont aining the alpha furanose, and a detectable amount of the aldehyde modification. There is a small but measurable displacement of equilibrium with change in temperature. The mutarotation is exceptionally sensitive to basic catalysts and for this reason the point of minimum rate lies in the acid region at $\mathrm{pH} 3.1$.
\end{abstract}

\section{Introduction}

The uronic acids, although few in number, constitute an important group of naturally occurring carbohydrate derivatives whose chemistry has not been adequately developed. Of the 16 possible isomeric hexuronic acids, only 3 have been found in nature. D-Glucuronic acid occurs in animal tissues, and frequently plays an important role in detoxifying processes. D-Galacturonic acid occurs in nearly all land plants as the chief constituent of pectic substances. An isomeric substance, D-mannuronic acid, is the principal constituent of algin. This polysaccharide is derived from certain marine algae [1], ${ }^{1}$ especially from Macrocystis pyrifera, the giant kelp of the Pacific coast, as well as from other species existing on the Atlantic coast and elsewhere. Crystalline man-

\footnotetext{
1 Figures in brackets indicate the literature references at the end of this paper.
}

Ring Structure of Mannuronic Lactone

\section{Contents}

I. Introduction Page

II. Ring structure of mannuronic lactone . . . . . 44

1. Stereomeric factors ....... 44

2. Application of the bromine oxidation method......... 44

3. Bromine oxidation measurements . . 45

III. Mutarotation of mannuronic lactone . . . . 46

1. Changes in optical rotation . . . . 46

2. Initial rapid mutarotation . . . . 46

(a) Application of the first-order equation ...... 47

(b) Catalysis by hydrogen and hydroxyl ions ...... 47

(c) Influence of temperature on the reaction rates .... 48

(d) Influence of temperature on the equilibrium state. . . . 48

IV. Summary . . . . . . . . . . 49

V. References ............. 49 
nuronic lactone [2] and two crystalline modifications of D-mannuronic acid [3] have been prepared from alginic acid. The lactone of mannuronic acid exhibits a rapid mutarotation, indicating the establishment of an equilibrium between at least two modifications. It has been assumed that the crystalline substance has a bicyclic structure, consisting of a lactone ring and a sugar ring, but no attempt has been made to assign a definite structure to the sugar entity.

\section{Ring Structure of Mannuronic Lactone}

\section{Stereomeric Factors}

A careful inspection of the structural models for mannuronic lactone reveals that it can readily form a bicyclic structure having two six-membered rings or two five-membered rings. The stereomeric relationship of the groups, however, is such that a compound having both a five- and a six-membered ring is highly improbable and may be eliminated from consideration. Thus the aldose function of a mannuronic gamma lactone can exist only in the open-chain form or in the furanoid form, whereas the aldose function of a mannuronic delta lactone can exist only in the open-chain form or in the pyranoid form. Ordinarily, gamma lactones are much more stable to hydrolysis than delta lactones, but there are exceptions to this general rule. For instance, Haworth, Jackson, Owen, and Smith [4] have pointed out that the presence of an anhydro ring in a sugar structure may affect in marked degree the relative stability of the pyranose and furanose modifications. Likewise Smith [5] has found that the dilactone of glucosaccharic acid, presumably having the 1,4-3,6-bicyclic structure, is hydrolyzed rapidly by water, and has suggested that the sensitivity to hydrolysis is due in part to the strain caused by the interlocking rings. Inasmuch as mannuronic lactone is relatively stable to hydrolysis, it might be expected to have the gamma lactone structure, but obviously this structure cannot be assigned merely from the stability of the compound to hydrolysis.

\section{Application of the Bromine Oxidation Method}

In previous publications from this Bureau [6] it has been shown that the ring forms of the sugars are oxidized by bromine water directly to lactones without the intermediate formation of the free acids. It has been shown also [7] that the two pyranose modifications of galacturonic acid on bromine oxidation yield an optically active lactone of mucic acid, a fact which demonstrates that the pyranose ring remains intact during the oxidation. ${ }^{2}$ Thus the bromine oxidation method is applicable to the study of the ring structure of the uronic acids.

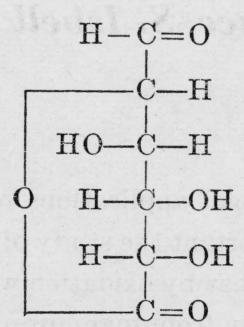

I

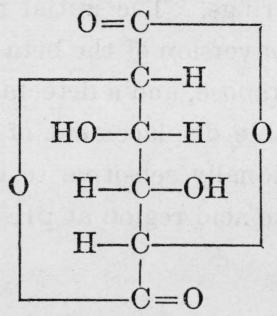

IV

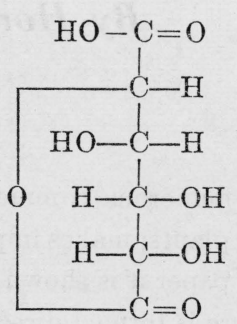

II

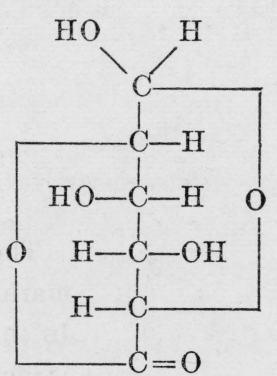

III

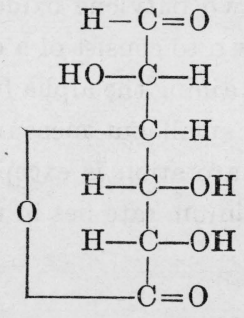

$\mathrm{V}$

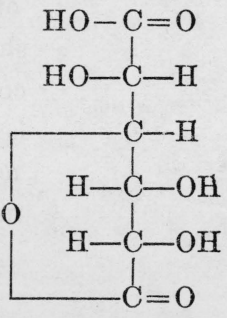

VI

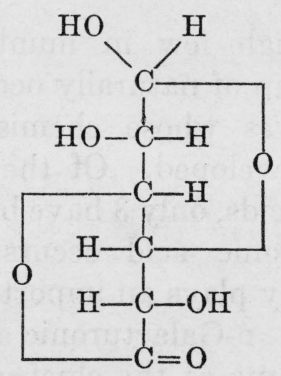

VII

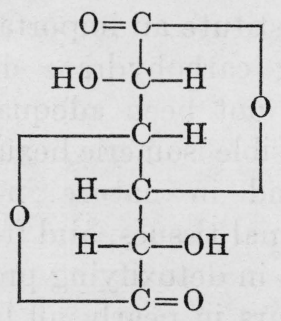

VIII
If mannuronic lactone had a delta lactone structure, oxidation of the aldehyde form I would yield the unknown delta monolactone of mannosaccharic acid II, and oxidation of the pyranose form III would yield the unknown delta dilactone

\footnotetext{
If the pyranose ring were ruptured before oxidation, the two carboxyl groups of the mucic acid, a meso compound, would have equal opportunity to form a lactone, and a racemic mixture would result.
} 
IV. If mannuronic lactone had a gamma lactone structure, the aldehyde form $\mathrm{V}$ would yield the unknown gamma monolactone of mannosaccharic acid VI, and the furanoid form VII would yield the known gamma dilactone VIII. The results described in this paper show that the oxidation product of mannuronic lactone is in fact the mannosaccharic gamma dilactone VIII, whose structure has been well established [8]. Hence mannuronic lactone has a furanoid structure for the sugar ring and the gamma structure for the lactone ring as represented in formula VII.

\section{Bromine Oxidation Measurements}

The formation of mannosaccharic dilactone from mannuronic lactone was studied by oxidation of the latter substance under conditions essentially the same as those previously used for the study of the ring structure of the sugars [9]. A 0.5-g sample of crystalline mannuronic lactone was added to a buffered solution prepared by the addition of $2 \mathrm{ml}$ of bromine to an ice-cold mixture containing $2 \mathrm{~g}$ of barium carbonate in $25 \mathrm{ml}$ of water saturated with carbon dioxide and contained in a glass-stoppered flask. The mixture was cooled in ice and kept in agitation by shaking. Time was measured beginning with the addition of the crystalline lactone to the bromine solution. After 5 minutes at $0^{\circ} \mathrm{C}$ a portion of the mixture was filtered. The temperature of the filtrate was raised to and kept at $20^{\circ} \mathrm{C}$. From time to time the optical rotations were read in a $1-\mathrm{dm}$ tube with a Bates saccharimeter. These values are reported in the second column of table 1.

TABLE 1.-Comparison of the optical rotation of the product obtained by bromine oxidation of mannuronic lactone with the optical rotation of mannosaccharic dilactone under like conditions.

\begin{tabular}{|c|c|c|}
\hline \multirow{2}{*}{ Time } & \multicolumn{2}{|c|}{ Solution obtained from- } \\
\cline { 2 - 3 } & $\begin{array}{c}\text { Mannuronic } \\
\text { lactone }\end{array}$ & $\begin{array}{c}\text { Mannosaccharic } \\
\text { dilactone }\end{array}$ \\
\hline Minutes & $\circ S$ & $\circ S$ \\
8 & +10.6 & $-\cdots$ \\
15 & +10.6 & +10.9 \\
45 & +10.5 & +10.6 \\
180 & +10.2 & +10.4 \\
1,320 & +8.6 & +8.8 \\
\hline
\end{tabular}

To establish the identity of the oxidation product, the optical rotations were compared with those of an equivalent amount of mannosaccharic dilactone that had been treated in the same manner. The optical rotations, recorded in table 1 , show that both solutions exhibit mutarotation, and that the values are in substantial agreement at all times. The mutarotation is undoubtedly caused by hydrolysis of the dilactone. As delta and gamma lactones differ markedly in sensitivity to hydrolysis, the agreement of the two solutions in respect to this property precludes the possibility of a delta lactone structure, and shows that the oxidation product of mannuronic lactone is indeed the known mannosaccharic dilactone having the gamma lactone structure.

The initial rotations of both solutions correspond to specific rotations somewhat lower than the recorded specific rotation of mannosaccharic dilactone. The difference presumably arises from the fact that a small part of the oxidation product and of the dilactone was converted to the barium salt.

The direction of the change in optical rotation is particularly significant. Rehorst [10] found that the specific rotation of a solution of mannosaccharic dilactone decreases from $+203.1^{\circ}$ in 47 minutes to $+69.5^{\circ}$ in 24 days, whereas that of a solution of mannosaccharic acid increases from $+3.6^{\circ}$ in 3 minutes to $+48.7^{\circ}$ in 27 days. The latter values expressed on the weight of the dilactone are $+4.3^{\circ}$ and $+58.8^{\circ}$. Thus the specific rotation of the equilibrium mixture lies between $+69.5^{\circ}$ and $+58.8^{\circ}$. The decrease in optical rotation of the solution from mannuronic lactone shows that mannosaccharic dilactone is present immediately after oxidation in excess of the equilibrium proportion, and must have been formed without the intermediate production of the free acid. It follows that there was no alteration in ring structure during oxidation and that mannuronic lactone has a bicyclic structure like that of mannosaccharic dilactone.

To confirm the conclusion that mannosaccharic dilactone is formed by bromine oxidation of mannuronic lactone without the intermediate formation of free mannosaccharic acid, $5 \mathrm{~g}$ of mannuronic lactone was oxidized under the conditions described above, and the product was isolated. The oxidation was stopped after 5 minutes by removal of the bromine with a mixture of 1 part of linseed oil and 2 parts of benzene. The solution was filtered, and an aliquot corresponding to four-fifths of the material was concentrated in vacuum at a temperature of $40^{\circ} \mathrm{C}$ to a semisolid crystalline massecuite, which was then extracted 
with a total of $200 \mathrm{ml}$ of acetone. The extract was filtered and evaporated in a stream of dry air. The residue, $2.32 \mathrm{~g}$, corresponding to a 59-percent yield, crystallized in slender needles. After one crystallization from ethyl alcohol, $[\alpha]_{\mathrm{D}}^{20}=+200.0^{\circ}$ (15 minutes. Water, $c=1.5$ ). For a sample of pure mannosaccharic dilactone prepared in this laboratory, $[\alpha]_{\mathrm{D}}^{20}=+201.1^{\circ}$ (15 minutes. Water, $\mathrm{c}=1.5)$.
Inasmuch as the oxidation was conducted in the presence of barium carbonate, a basic substance, and the product was extracted with acetone without previous acidification, the formation of the free mannosaccharic acid could not have been an intermediate step in obtaining the dilactone as described above.

\section{Mutarotation of Mannuronic Lactone}

\section{Changes in Optical Rotation}

Nelson and Cretcher [2], who first prepared mannuronic Iactone, reported a mutarotation from $+79.9^{\circ}$ at 3 minutes to a maximum of $+93.8^{\circ}$ in 28 minutes, followed by a slow decrease over a period of days. After 864 hours the solution had a specific rotation of $+61.8^{\circ}$ and contained 39.1 percent of acid and 60.9 percent of lactone. The authors state that "the rapid initial increase is probably due to attainment of equilibrium between the $\alpha$ and $\beta$ forms of the aldehyde, and the subsequent decrease is due to the formation of mannuronic acid." Subsequently, Ault, Haworth, and Hirst [11] reported an optical rotation of $+95^{\circ}$, with no mutarotation, whereas Stacey and Wilson [12] reported a value of $+50^{\circ}$, increasing to $+94^{\circ}$ in 30 minutes. The measurements obtained in the present investigation (table 2) show an

TABLE 2.-Mutarotation of mannuronic lactone in water at $20^{\circ} \mathrm{C}$

$4 \mathrm{~g}$ per $100 \mathrm{ml}$, read in a $2-\mathrm{dm}$ tube.

o $S=-7.86 \times 10^{-0.125}+21.02$.

$[\alpha]_{\mathrm{D}}^{20}=-34.5 \times 10^{-0.125 t}+92.2$.

$[\alpha]_{\mathrm{D}}^{20}=+57.7$ (initial), +92.2 (maximum).

\begin{tabular}{|c|c|c|c|c|c|}
\hline Time & $\begin{array}{l}\text { Observed } \\
\text { reading }\end{array}$ & $m$ & Time & $\begin{array}{l}\text { Observed } \\
\text { reading }\end{array}$ & $m$ \\
\hline Minutes & $\circ S$ & & Minutes & ${ }^{\circ} S$ & \\
\hline 0 & - n. & $\ldots$ & 4.4 & 18.83 & 0.126 \\
\hline 0 & 14.78 & ..... & 5.1 & 19.21 & .125 \\
\hline 1.2 & 15.45 & 0.123 & 6.0 & 19.60 & .124 \\
\hline 1.7 & 16.25 & .130 & 7.1 & 19.97 & .123 \\
\hline 2.0 & 16.55 & .121 & 8.5 & 20.27 & .120 \\
\hline 2.2 & 16.90 & .129 & 9.7 & 20.53 & .124 \\
\hline 2.5 & 17.25 & .129 & 60.0 & $\begin{array}{c}21.02 \\
\text { (maximum) }\end{array}$ & $\ldots$ \\
\hline$A v$ & & & & & 0.125 \\
\hline
\end{tabular}

a This equation is converted to a specific-rotation basis by multiplying by the ratio of the maximum specific rotation (measured on a separate sample) to the observed maximum rotation in sugar degrees. The method for deriving the equation and calculating the initial rotation is given on page 156 of reference [9]. initial rotation of $+57.7^{\circ}$ and a rapid increase to a maximum of $92.2^{\circ}$ in about half an hour. As noted by Nelson and Cretcher, the subsequent decrease in optical rotation is caused by cleavage of the lactone rather than by a change in the sugar function and consequently it will not be discussed in this paper.

\section{Initial Rapid Mutarotation}

As mannuronic lactone has a bicyclic structure with a furanose ring and a gamma lactone ring, the initial rapid mutarotation might involve the alpha and beta furanose modifications and the openchain modification of the sugar function or hydrolysis of the lactone ring. However, it has been shown [2] that the slow mutarotation is due to the hydrolysis of the lactone ring, and hence the rapid mutarotation must be associated with changes in the sugar function. When the material is treated with Schiff's aldehyde reagent, a pink color gradually develops. The intensity of the color is greater than that obtained with galacturonic acid or with mannose. However, as the color does not appear immediately, but only after an interval of at least 5 minutes, it may be assumed that the crystalline material is not the aldehyde, but that some of the aldehyde is formed by the mutarotation reaction. The aldehyde modification of mannuronic lactone differs from mannonic gamma lactone only in that it contains a $\mathrm{CHO}$ group in place of the terminal $\mathrm{CH}_{2} \mathrm{OH}$ group, and consequently it may be assumed that the specific rotation of the aldehyde form does not differ widely from the specific rotation of mannonic gamma lactone $(+51)$. During the mutarotation, the specific rotation increases from +58 to +92. This requires the formation of a substance having an optical rotation of at least +92 , a value considerably larger than that anticipated for the aldehyde. Undoubtedly, the substance is the 
alpha furanose modification. Thus the reaction involves establishment of equilibrium between the alpha and beta furanose modifications and the aldehyde. As already pointed out, the presence of a gamma lactone ring precludes the existence of mannuronic lactone in the pyranoid form. As an equilibrium solution of mannose does not contain an appreciable quantity of the aldehyde form, the presence of the aldehyde in the equilibrium solution of mannuronic lactone shows that the lactone ring enhances the tendency of the sugar to exist in the aldehyde form.

\section{(a) Application of the first-order equation}

As shown by the values given in table 2, the rapid mutarotation follows the usual first-order equation:

$$
m=\frac{1}{t} \log \frac{r_{o}-r_{\infty}}{r_{t}-r_{\infty}},
$$

in which $r_{t}$ is the rotation at time $t, r \infty$ is the maximum rotation, $r_{0}$ is the rotation at the beginning of the time period $t$, and $m$ is the mutarotation constant. The observed value for the mutarotation constant of mannuronic lactone at $20^{\circ} \mathrm{C}$ $(0.125)$ is much higher than the constant (0.0148) for the interconversion of the pyranose modifications of galacturonic acid [7], but it does not differ widely from that $(0.16)$ found for the rapid mutarotation reaction of galacturonic acid hydrate, which was ascribed to a pyranose-furanose interconversion.

\section{(b) Catalysis by hydrogen and hydroxyl ions}

It has been established [13] that the mutarotation constants for sugars in solutions of varying acidity may be represented by an equation of the type:

$$
m=A+B[\mathrm{H}]+C[\mathrm{OH}],
$$

in which $A$ represents the catalytic effect of the solvent, $B[\mathrm{H}]$ the catalytic effect of the hydrogen ion, and $C[\mathrm{OH}]$ the catalytic effect of the hydroxyl ion. Some values obtained for the mutarotation constant of mannuronic lactone at various acidities are given in table 3 . The following equation, which represents the effect of hydrogen and hydroxyl ions on the mutarotation constant of mannuronic lactone at $1.2^{\circ} \mathrm{C}$, was calculated from three simultaneous equations, using the values at $\mathrm{pH} 1.0,3.0$, and 4.9:

$$
m=0.0057+0.59[\mathrm{H}]+260,000,000[\mathrm{OH}] \text {. }
$$

\begin{tabular}{|c|c|c|}
\hline Solution & $\begin{array}{l}\text { Mutarota- } \\
\text { tion con- } \\
\text { stant }\end{array}$ & $\underset{\text { (measured) }}{\mathrm{pH}}$ \\
\hline $0.1 \mathrm{~N} \mathrm{HCl}$ & 0.065 & 1.0 \\
\hline $0.01 \mathrm{NHCl}$ & .0115 & 2.0 \\
\hline $0.001 N \mathrm{HCl}$ & .0066 & 3.0 \\
\hline Water & .0106 & 4.0 \\
\hline Nitrophenol buffer ${ }^{\mathbf{a}}$ & .0318 & 4.9 \\
\hline
\end{tabular}

TABLE 3.-Mutarotation constants for mannuronic lactone at $1.2^{\circ} \mathrm{C}$ in aqueous solutions of various acidities

a This buffer solution consisted of 1 part of 0 -nitrophenol solution saturated at $20^{\circ} \mathrm{C}$ and 1 part of water. Sodium hydroxide was added to the extent of 0.0004 equivalent per liter.

The constant passes through a minimum (0.0066) at a hydrogen concentration of $7.4 \times 10^{-4}$ (pH 3.13), at which point the catalytic effect of the solvent is approximately six times the combined catalytic effect of the hydrogen and hydroxyl ions. The mutarotation constants at $20^{\circ} \mathrm{C}$ for levulose and glucose are reported to show minima at $\mathrm{pH} 4.4$ and 4.6, respectively [14]. It is of interest to compare the relative sensitivities of the mutarotations of mannuronic lactone, levulose, and glucose to the catalytic effect of hydrogen and hydroxyl ions. The data for mannuronic lactone is not directly comparable with that previously reported for levulose and glucose. A qualitative comparison can be made, however, by taking the minimum value of the constant for each substance as unity. This eliminates the catalytic effect of the solvent, the effect of the difference in temperature under which the measurements were made, and the large difference in minimum rates. A comparison of this character is shown in figure 1.

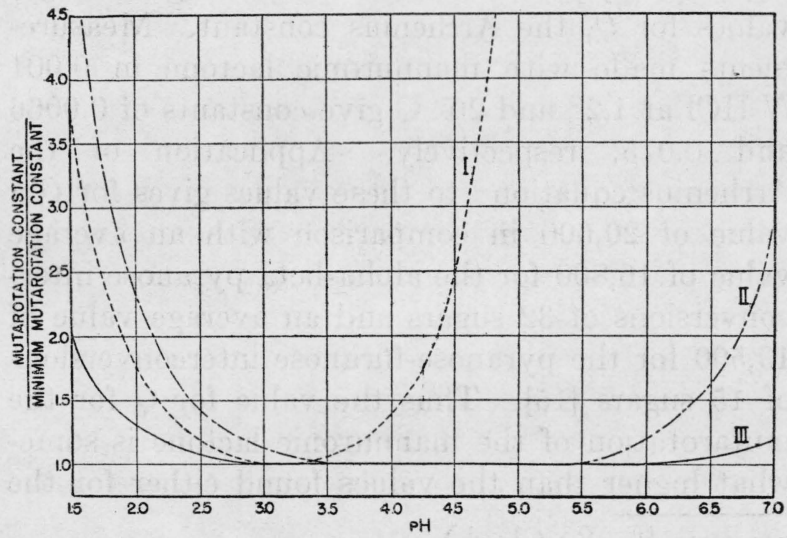

FIgURE 1.-Variation of the mutarotation constants with acidity.

I. Mannuronic lactone, an $\alpha-\beta$-furanose interconversion.

II. Levulose, a pyranose-furanose interconversion. Data from reference [14].

III. Glucose, an $\alpha$ - $\beta$-pyranose interconversion. Data from reference [14] 
The mutarotation of mannuronic lactone, an alpha-beta furanose interconversion, is more sensitive to the catalytic action of hydrogen ion than the mutarotation of glucose, an alpha-beta pyranose interconversion, and less sensitive than that of levulose, a pyranose-furanose interconversion. With respect to the hydroxyl ion, the mutarotation of mannuronic lactone is far more sensitive than the mutarotations of either glucose or levulose. In fact, the catalytic effect of the hydroxyl ion is so strong that at $\mathrm{pH} 7$ the mutarotation is too rapid to be detected even at $1.2^{\circ} \mathrm{C}$. The high sensitivity to the hydroxyl ion may be a characteristic property of alpha-beta furanose interconversions, but as the substance has a bicyclic structure, its mutarotation may not be typical of alpha-beta furanose interconversions in general. The long horizontal section of the glucose curve indicates that in the $\mathrm{pH}$ range from 2 to 7 the predominating rate-determining factor is the catalytic effect of the water, and that the specific catalytic effect of the hydrogen and hydroxyl ions is relatively small. The almost complete absence of a horizontal section in the curve for mannuronic lactone indicates that at all acidities the hydrogen and hydroxyl ions influence the reaction rate substantially.

\section{(c) Influence of temperature on the reaction rates}

Another point of distinction between reactions of the alpha-beta pyranose type and reactions of the pyranose-furanose type is that the former have higher temperature coefficients and give larger values for $Q$, the Arrhenius constant. Measurements made with mannuronic lactone in 0.001 $N \mathrm{HCl}$ at $1.2^{\circ}$ and $20^{\circ} \mathrm{C}$ give constants of 0.0066 and 0.075 , respectively. Application of the Arrhenius equation ${ }^{3}$ to these values gives for $Q$ a value of 20,600 in comparison with an average value of 16,800 for the alpha-beta pyranose interconversions of 32 sugars and an average value of 13,500 for the pyranose-furanose interconversions of 15 sugars [15]. Thus the value for $Q$ for the mutarotation of the mannuronic lactone is somewhat higher than the values found either for the

$$
\text { s } 2.3026 \log \frac{k_{1}}{k_{2}}=\frac{Q}{1.9864}\left(\frac{1}{T_{2}}-\frac{1}{T_{1}}\right) \text {. }
$$

alpha-beta pyranose interconversions or for the pyranose-furanose interconversions of the sugars.

\section{(d) Influence of temperature on the equilib- rium rates}

Reactions of the pyranose-furanose type also differ from those of the alpha-beta pyranose type in that they usually involve larger energy changes. If the energy change is small, as in the interconversion of the alpha and beta glucopyranoses, there is little displacement of the equilibrium with change in temperature. But if the energy change is high, as in the case of the pyranose-furanose interconversion of fructose, there is a considerable displacement of equilibrium with change of temperature. As shown by the data of table 4, when the temperature of a solution of mannuronic, lactone of maximum rotation is suddenly lowered there is a small but measureable mutarotation that takes place at a rate substantially the same as that for a solution freshly prepared from crystalline mannuronic lactone. The optical rotation decreases, and hence the displacement of equilibrium on cooling is in favor of a less dextrorotatory substance, presumably the beta furanose or the aldehyde modification. As the change in the equilibrium with change in temperature appears to be small, it seems probable that the heat of reaction is also small.

TABLE 4.- Thermal mutarotation of a solution of mannuronic lactone in $0.001 \mathrm{~N} \mathrm{HCl}$ after cooling from $20^{\circ}$ to $1.0^{\circ} \mathrm{C}$

[10 $\mathrm{g}$ of mannuronic lactone per $100 \mathrm{ml}$ of solution read in a 2-dm tube]

\begin{tabular}{|c|c|c|}
\hline $\begin{array}{c}\text { Time after } \\
\text { cooling }\end{array}$ & $\begin{array}{c}\text { Observed } \\
\text { reading }\end{array}$ & $\begin{array}{c}\text { Mutarotation } \\
\text { constant a }\end{array}$ \\
\hline & & \\
Minutes & $\circ$ & $m$ \\
4.3 & 50.28 & $\ldots . .10$ \\
8.5 & 50.20 & 0.010 \\
15.0 & 50.06 & .012 \\
24.7 & 49.96 & .010 \\
43.4 & 49.81 & .009 \\
59.5 & 49.73 & .008 \\
103.6 & 49.58 & .007 \\
Constant & 49.41 & $\ldots \ldots . . . . .$. \\
& & \\
\hline
\end{tabular}

- As the values given in this column are based on small changes in optical rotation, the experimental error is large, and the agreement with the mutarotation constant of the lactone in $0.001 \mathrm{NHCl}, 0.0066$, is as close as could be expected. 


\section{Summary}

Oxidation of mannuronic lactone with bromine water under conditions that preclude rupture of the sugar ring gives the dilactone of mannosaccharic acid that is known to have a 1,4-3,6bicyclic structure. The production of this substance shows that mannuronic lactone is a bicyclic compound containing a 1,4-furanose ring and a 3,6-lactone ring.

The mutarotation of mannuronic lactone is characterized by two reactions, a rapid conversion of the beta furanose modification to a mixture containing the alpha furanose, and the aldehyde modification, and a slow hydrolysis of the lactone ring. The mutarotation of the sugar component in water at $20^{\circ} \mathrm{C}$ and at $\mathrm{pH} 4.0$ follows the equation:

$$
[\alpha]_{\mathrm{D}}^{20}=-34.5 \times 10^{-0.125 t}+92.2 .
$$

$[\alpha]_{D}^{20}=+57.7^{\circ}$ initially, and $+92.2^{\circ}$ after 30 minutes (maximum). The rate of mutarotation is considerably higher than that found previously for the alpha-beta pyranose interconversion of galacturonic acid, but it does not differ widely from the rate attributed to the pyranose-furanose intercon- version of this substance. The mutarotation rate does not change markedly in the range from $\mathrm{pH} 2$ to $\mathrm{pH} 4$, but in more strongly acid or alkaline solution, the rate rises sharply. The catalytic influence of the hydrogen and hydroxyl ions on the mutarotation constant at $1.2^{\circ} \mathrm{C}$ may be represented by the equation:

$$
m=0.0057+0.59[\mathrm{H}]+260,000,000[\mathrm{OH}] .
$$

The marked sensitivity of the substance to the catalytic effect of hydroxyl ions causes the point of minimum rate of mutarotation to lie far in the acid range ( $\mathrm{pH}$ 3.1). Mutarotation measurements at $1.2^{\circ} \mathrm{C}$ and $20^{\circ} \mathrm{C}$ in $0.001 \mathrm{~N} \mathrm{HCl}$ give constants of 0.0066 and 0.075 , respectively, corresponding to a heat of activation of 20,600 calories. There is a small but measurable thermal mutarotation when the temperature of a solution of mannuronic lactone of maximum rotation is suddenly lowered. The rate of this mutarotation is substantially the same as that of a solution freshly prepared from crystalline mannuronic lactone. The direction of the change indicates the production of either the beta furanose or the aldehyde modification.

\section{References}

[1] A. G. Norman, The biochemistry of cellulose, the polyuronides, lignin, etc., pp. 142-146 (Oxford University Press, 1937).

[2a] W. L. Nelson and L. H. Cretcher, J. Am. Chem. Soc. 54, 3409 (1932).

[2b] E. Schoeffel and K. P. Link, J. Biol. Chem. 95, 213 (1932).

[3] E. Schoeffel and K. P. Link, J. Biol. Chem. 100, 397 (1933).

[4] W. N. Haworth, J. Jackson, and F. Smith, J. Chem. Soc. 1940, 620; W. N. Haworth, L. N. Owen, and F. Smith, J. Chem. Soc. 1941, 88.

[5] F. Smith, J. Chem. Soc. 1944, 633.

[6] H. S. Isbell and C. S. Hudson, BS J. Research 8, 327 (1932) RP418; H. S. Isbell, BS J. Research 8, 615 (1932) RP441.

[7] H. S. Isbell and H. L. Frush, J. Research NBS 31, 33 (1943) RP1549.
[8] O. Th. Schmidt and T. H. Kraft, Ber. deut. chem. Ges. 74, 33 (1941); D. Heslop and F. Smith, J. Chem. Soc. 1944, 574.

[9] H. S. Isbell and W. W. Pigman, J. Research NBS 18, 141 (1937) RP969.

[10] K. Rehorst, Ber. deut. chem. Ges. 65, 1476 (1932).

[11] R. G. Ault, W. N. Haworth, and E. L. Hirst, J. Chem. Soc. $1935,517$.

[12] M. Stacey and P. I. Wilson, J. Chem. Soc. 1944, 588.

[13] C. S. Hudson, J. Am. Chem. Soc. 29, 1572 (1907).

[14] H. S. Isbell and W. W. Pigman, J. Research NBS 20, 773 (1938) RP1104.

[15] F. J. Bates and associates, Polarimetry, saccharimetry, and the sugars, Circular National Bureau of Standards C440 (1942).

Washington, May 1, 1946. 